\title{
Expansions of Jacobian Elliptic Functions in Powers of the Modulus
}

\author{
By Y. K. Lin* and F. A. Lee
}

The Maclaurin expansions for Jacobian elliptic functions in the neighborhood of zero modulus (to be called the $k$-expansions) are given herein. Since the absolute value of modulus $k$ can be taken as smaller than one without loss of generality, the $k$ expansions in most cases converge faster than similar expansions in the neighborhood of the zero argument [1] (to be called the $u$-expansions). The relative accuracies of the two types of expansions will be illustrated numerically for several pairs of $k$ and $u$ values and by taking equal numbers of terms in the computation. The $k$ expansions also provide for the information, in a fundamental way, as to how the Jacobian functions vary with the change of modulus. The information may be useful in the study of damped non-linear vibrations.

The basic Jacobian function $s n\left(u, k^{2}\right)$ is related to its argument, $u$, and modulus, $k$, as the following:

$$
u=\int_{0}^{s n(u, k)} \frac{d t}{\sqrt{1-t^{2}} \sqrt{1-k^{2} t^{2}}}=\int_{0}^{s n(u, \phi)} \frac{d t}{\sqrt{1-t^{2}} \sqrt{1-\phi t^{2}}} .
$$

By successive differentiations one obtains

$$
\left\{\begin{aligned}
& \frac{\partial s n}{\partial \phi}=- c n \cdot d n \int_{0}^{s n} \frac{\left(t^{2} / 2\right) d t}{\sqrt{\left(1-t^{2}\right)\left(1-\phi t^{2}\right)^{3}}} \\
& \frac{\partial^{2} s n}{\partial \phi^{2}}=- c n \cdot d n\left[\int_{0}^{s n} \frac{1 \cdot 3\left(t^{2} / 2\right)^{2} d t}{\sqrt{\left(1-t^{2}\right)\left(1-\phi t^{2}\right)^{5}}}\right. \\
&\left.\quad+\frac{s n^{2}}{2 c n \cdot d n^{3}} \cdot \frac{\partial s n}{\partial \phi}+\frac{\partial s n}{\partial \phi} \cdot \frac{\partial}{\partial \phi}\left(\frac{1}{c n \cdot d n}\right)\right] \\
& \frac{\partial^{m} s n}{\partial \phi^{m}}=-c n \cdot d n\left[\int_{0}^{s n} \frac{1 \cdot 3 \cdot 5 \cdots(2 m-1)\left(t^{2} / 2\right)^{m}}{\sqrt{\left(1-t^{2}\right)\left(1-\phi t^{2}\right)^{2 m+1}}} d t\right. \\
&+\frac{\partial s n}{\partial \phi}\left[\frac{1 \cdot 3 \cdot 5 \cdots(2 m-3)\left(s n^{2} / 2\right)^{m-1}}{c n \cdot d n^{2 m-1}}\right. \\
&\left.+\frac{\partial}{\partial \phi}\left(\frac{1 \cdot 3 \cdot 5 \cdots(2 m-5)\left(s n^{2} / 2\right)^{m-2}}{c n \cdot d n^{2 m-3}}\right)+\cdots+\frac{\partial^{m-1}}{\partial \phi^{m-1}}\left(\frac{1}{c n \cdot d n}\right)\right] \\
&+\frac{\partial^{2} s n}{\partial \phi^{2}}\left[\frac{1 \cdot 3 \cdot 5 \cdots(2 m-5)\left(s n^{2} / 2\right)^{m-2}}{c n \cdot d n^{2 m-3}}\right. \\
&+2 \frac{\partial}{\partial \phi}\left(\frac{1 \cdot 3 \cdot 5 \cdots(2 m-7)\left(s n^{2} / 2\right)^{m-3}}{c n \cdot d n^{2 m-5}}\right) \\
&\left.+\cdots+(m-1) \frac{\partial^{m-2}}{\partial \phi^{m-2}}\left(\frac{1}{c n \cdot d n}\right)\right]+\cdots \\
&\left.+\frac{\partial^{m-1} s n}{\partial \phi^{m-1}}\left[(m-1) \frac{\partial}{\partial \phi}\left(\frac{1}{c n \cdot d n}\right)\right]\right\} \cdot
\end{aligned}\right.
$$

Received July 24, 1961.

* Consultant, and Assistant Professor of Aeronautical Engineering, University of Illinois. 
It follows upon evaluating these derivatives at $\phi=0$ and noting that $\phi=k^{2}$,

$$
\begin{aligned}
\operatorname{sn}\left(u, k^{2}\right)=\sin u & -\frac{\cos u}{4}\left(u-\frac{\sin 2 u}{2}\right) k^{2} \\
-\frac{\cos u}{4} & {\left[\frac{3}{4}\left(\frac{3 u}{2}-\sin 2 u+\frac{\sin 4 u}{8}\right)-\sin ^{2} u\left(u-\frac{\sin 2 u}{2}\right)\right.} \\
& \left.+\frac{\tan u}{4}\left(u-\frac{\sin 2 u}{2}\right)^{2}\right] \frac{k^{4}}{2 !}-\cdots+\left\{\frac{\partial^{m} s n}{\partial \phi^{m}}\right\}_{\phi=0} \cdot \frac{k^{2 m}}{m !}-\cdots
\end{aligned}
$$

Expansions for other Jacobian functions can be generated from the relations between these functions and $s n$. The results are given explicitly up to $k^{4}$ in the following:

$$
\begin{aligned}
& c n\left(u, k^{2}\right)=\cos u+\frac{\sin u}{4}\left(u-\frac{\sin 2 u}{2}\right) k^{2}-\left\{\frac{\cos u}{16}\left(u-\frac{\sin 2 u}{2}\right)^{2}\right. \\
& \left.+\frac{\sin u}{4}\left[\sin ^{2} u\left(u-\frac{\sin 2 u}{2}\right)-\frac{3}{4}\left(\frac{3}{2} u-\sin 2 u+\frac{\sin 4 u}{8}\right)\right]\right\} \frac{k^{4}}{2 !}+\cdots \text {. } \\
& d n\left(u, k^{2}\right)=1-\frac{1}{2}\left(\sin ^{2} u\right) k^{2}+\left(\frac{u \sin 2 u}{4}-\frac{\sin ^{2} 2 u}{8}-\frac{1}{4} \sin ^{4} u\right) \frac{k^{4}}{2 !}+\cdots \\
& n s\left(u, k^{2}\right)=\csc u+\csc ^{2} u\left(\frac{\cos u}{4}\right)\left(u-\frac{\sin 2 u}{2}\right) k^{2}+\left[-\frac{\cos u}{4}\left(u-\frac{\sin 2 u}{2}\right)\right. \\
& -\frac{3}{16} \cos u \csc ^{2} u\left(\frac{3}{2} u-\sin 2 u+\frac{\sin 4 u}{8}\right)
\end{aligned}
$$

$$
\left.+\frac{1}{16} \csc u\left(\csc ^{2} u+\cot ^{2} u\right)\left(u-\frac{\sin 2 u}{2}\right)^{2}\right] \frac{k^{4}}{2 !}+\cdots
$$

$$
\begin{aligned}
\operatorname{cs}\left(u, k^{2}\right) & =\cot u+\frac{\csc ^{2} u}{4}\left(u-\frac{\sin 2 u}{2}\right) k^{2}+\left[-\frac{1}{8}\left(u-\frac{\sin 2 u}{2}\right)^{2} \cot u \csc ^{2} u\right. \\
& \left.+\frac{3}{16} \csc ^{2} u\left(\frac{3}{2} u-\sin 2 u+\frac{\sin 4 u}{8}\right)-\frac{1}{4}\left(u-\frac{\sin 2 u}{2}\right)\right] \frac{k^{4}}{2 !}+\cdots
\end{aligned}
$$$$
d s\left(u, k^{2}\right)=\csc u+\left[\frac{1}{4} \cot u \csc u\left(u-\frac{\sin 2 u}{2}\right)-\frac{1}{2} \sin u\right] k^{2}
$$

$$
\begin{aligned}
+\frac{1}{4}\left[-\sin ^{3} u+\right. & \left(u-\frac{\sin u}{2}\right)^{2}\left(\frac{\csc u}{4}\right)\left(\csc ^{2} u+\cot ^{2} u\right) \\
& \left.\quad+\frac{3}{4} \cot u \csc u\left(\frac{3}{2} u-\sin 2 u+\frac{\sin 4 u}{8}\right)\right] \frac{k^{4}}{2 !}+\cdots \\
\operatorname{sc}\left(u, k^{2}\right)= & \tan u-\frac{1}{4} \sec ^{2} u\left(u-\frac{\sin 2 u}{2}\right) k^{2} \\
& -\frac{1}{4}\left[\frac{3}{8}\left(1+\sec ^{2} u\right)\left(\frac{3}{2} u-\sin 2 u+\frac{\sin 4 u}{8}\right)\right. \\
& \left.-\left(u-\frac{\sin 2 u}{2}\right) \tan ^{2} u-\frac{1}{2}\left(u-\frac{\sin 2 u}{2}\right)^{2} \tan u \sec ^{2} u\right] \frac{k^{4}}{2 !}-\cdots
\end{aligned}
$$


(10)

$$
\begin{aligned}
d c\left(u, k^{2}\right)=\sec u-\frac{1}{4} \tan u\left[2 \sin u+\sec u\left(u-\frac{\sin 2 u}{2}\right)\right] k^{2} \\
+\left[\frac{\sec u}{4}\left(u \sin 2 u-\frac{\sin ^{2} 2 u}{2}-\sin ^{4} u\right)+\frac{1}{2} \tan ^{2} u \sin u\left(u-\frac{\sin 2 u}{2}\right)\right. \\
-\frac{3}{16} \sin u \sec ^{2} u\left(\frac{3}{2} u-\sin 2 u+\frac{\sin 4 u}{8}\right) \\
\left.\quad-\frac{1}{16} \sec u\left(u-\frac{\sin 2 u}{2}\right)^{2}\left(\tan ^{2} u+\sec ^{2} u\right)\right] \frac{k^{4}}{2 !}+\cdots
\end{aligned}
$$$$
\left.n c\left(u, k^{2}\right)\right)=\sec u-\frac{\tan u \sec u}{4}\left(u-\frac{\sin 2 u}{2}\right) k^{2}
$$

(11) $+\left[\left(\frac{1}{16} \sec u+\frac{1}{8} \tan ^{2} u \sec u\right)\left(u-\frac{\sin 2 u}{2}\right)^{2}+\frac{1}{4} \tan ^{2} u \sin u\left(u-\frac{\sin 2 u}{2}\right)\right.$

$$
\left.+\frac{3}{4} \sec ^{2} u\left(\frac{3}{2} u-\sin 2 u+\frac{\sin 4 u}{8}\right)\right] \frac{k^{4}}{2 !}+\cdots \text {. }
$$

\begin{tabular}{|c|c|c|c|c|c|c|c|c|c|c|c|}
\hline \multirow{3}{*}{$u$} & \multirow{3}{*}{$\underset{k}{\operatorname{Sin}^{-1}}$} & \multicolumn{5}{|c|}{$s n$} & \multicolumn{5}{|c|}{$c n$} \\
\hline & & \multicolumn{2}{|c|}{$k$-Series } & \multicolumn{2}{|c|}{$u$-Series } & \multirow[b]{2}{*}{$\begin{array}{l}\text { Exact } \\
\text { Value }\end{array}$} & \multicolumn{2}{|c|}{$k$-Series } & \multicolumn{2}{|c|}{$u$-Series } & \multirow[b]{2}{*}{$\begin{array}{l}\text { Exact } \\
\text { Value }\end{array}$} \\
\hline & & $\begin{array}{l}\text { Com- } \\
\text { puted } \\
\text { Value }\end{array}$ & $\begin{array}{c}\% \\
\text { Er- } \\
\text { ror }\end{array}$ & $\begin{array}{l}\text { Com- } \\
\text { puted } \\
\text { Value }\end{array}$ & $\begin{array}{c}\% \\
\text { Er- } \\
\text { ror }\end{array}$ & & $\begin{array}{l}\text { Com- } \\
\text { puted } \\
\text { Value }\end{array}$ & $\begin{array}{c}\% \\
\text { Er- } \\
\text { ror }\end{array}$ & $\begin{array}{l}\text { Com- } \\
\text { puted } \\
\text { Value }\end{array}$ & $\begin{array}{c}\% \\
\text { Error }\end{array}$ & \\
\hline $\begin{array}{r}.1745 \\
.1754 \\
.7016 \\
.9401 \\
1.1424 \\
1.3372 \\
1.5738\end{array}$ & $\begin{array}{r}5^{\circ} \\
80^{\circ} \\
15^{\circ} \\
50^{\circ} \\
45^{\circ} \\
40^{\circ} \\
5^{\circ}\end{array}$ & $\begin{array}{l}.17361 \\
.17365 \\
.64277 \\
.76597 \\
.86587 \\
.93964 \\
.99999\end{array}$ & $\begin{array}{l}.02 \\
0 \\
0 \\
.01 \\
.02 \\
.01 \\
0\end{array}$ & $\begin{array}{c}.17361 \\
.17365 \\
.64294 \\
.77886 \\
.90344 \\
1.02184 \\
1.000821\end{array}$ & $\begin{array}{c}.02 \\
0 \\
.02 \\
1.67 \\
4.32 \\
8.74 \\
.82\end{array}$ & $\begin{array}{r}.17365 \\
.17365 \\
.64279 \\
.76604 \\
.86603 \\
.93969 \\
1.0000\end{array}$ & $\begin{array}{c}.98481 \\
.98481 \\
.76606 \\
.64282 \\
.50024 \\
.34250 \\
0\end{array}$ & $\begin{array}{l}0 \\
0 \\
0 \\
0 \\
.05 \\
.14 \\
0\end{array}$ & $\begin{array}{l}.98481 \\
.98654 \\
.76668 \\
.66704 \\
.56037 \\
.45935 \\
.02496\end{array}$ & $\begin{array}{r}0 \\
.18 \\
.08 \\
3.77 \\
12.07 \\
34.3\end{array}$ & $\begin{array}{l}.98481 \\
.98481 \\
.76604 \\
.64279 \\
.50000 \\
.34202 \\
0\end{array}$ \\
\hline
\end{tabular}

\begin{tabular}{|c|c|c|c|c|c|c|}
\hline \multirow{3}{*}{$u$} & \multirow{3}{*}{$\underset{k}{\operatorname{Sin}^{-1}}$} & \multicolumn{5}{|c|}{$d n$} \\
\hline & & \multicolumn{2}{|c|}{$k$-Series } & \multicolumn{2}{|c|}{$u$-Series } & \multirow[b]{2}{*}{$\begin{array}{l}\text { Exact } \\
\text { Value }\end{array}$} \\
\hline & & $\begin{array}{l}\text { Com- } \\
\text { puted } \\
\text { Value }\end{array}$ & $\begin{array}{l}\% \\
\text { Er- } \\
\text { ror }\end{array}$ & $\begin{array}{l}\text { Com- } \\
\text { puted } \\
\text { Value }\end{array}$ & $\begin{array}{l}\% \\
\text { Er- } \\
\text { ror }\end{array}$ & \\
\hline .1745 & $5^{\circ}$ & .99986 & 0 & .99988 & 0 & .99988 \\
\hline .1754 & $80^{\circ}$ & .98525 & 0 & .98698 & .17 & .98527 \\
\hline .7016 & $15^{\circ}$ & .98606 & 0 & .98626 & .02 & .98606 \\
\hline .9401 & $50^{\circ}$ & .80898 & .09 & .82828 & 2.29 & .80972 \\
\hline 1.1424 & $45^{\circ}$ & .79147 & .12 & .83341 & 5.42 & .79056 \\
\hline 1.3372 & $40^{\circ}$ & .80027 & .41 & .87352 & 9.61 & .79697 \\
\hline 1.5738 & $5^{\circ}$ & .99620 & 0 & .99837 & .22 & .99619 \\
\hline
\end{tabular}

(12) $n d\left(u, k^{2}\right)=1+\frac{\sin ^{2} u}{2} k^{2}-\frac{1}{4}\left[u \sin 2 u-\frac{\sin ^{2} 2 u}{2}-3 \sin ^{4} u\right] \frac{k^{4}}{2 !}+\cdots$.

TABLE 1

Computed Jacobian Elliptic Functions 


$$
\begin{aligned}
& c d\left(u, k^{2}\right)=\cos u+\frac{\sin u}{4}\left(u+\frac{\sin 2 u}{2}\right) k^{2}+\left[-\frac{\cos u}{16}\left(u-\frac{\sin 2 u}{2}\right)^{2}\right. \\
& +\frac{3}{16} \sin u\left(\frac{3}{2} u-\sin 2 u+\frac{\sin 4 u}{8}\right) \\
& \left.-\frac{\cos u}{4}\left(u \sin 2 u-\frac{\sin ^{2} 2 u}{2}-3 \sin ^{4} u\right)\right] \frac{k^{4}}{2 !}+\cdots . \\
& s d\left(u, k^{2}\right)=\sin u-\left[\frac{\cos u}{4}\left(u-\frac{\sin 2 u}{2}\right)-\frac{\sin ^{3} u}{2}\right] k^{2} \\
& -\left\{\frac{\cos u}{4}\left[\frac{3}{4}\left(\frac{3 u}{2}-\sin 2 u+\frac{\sin 4 u}{8}\right)+\frac{\tan u}{4}\left(u-\frac{\sin 2 u}{2}\right)^{2}\right]\right. \\
& \left.+\frac{\sin u}{4}\left(u \sin 2 u-\frac{\sin ^{2} 2 u}{2}-3 \sin ^{4} u\right)\right\} \frac{k^{4}}{2 !}-\cdots \text {. }
\end{aligned}
$$

Representative results computed for basic Jacobian functions using the $k$-series as well as the $u$-series are given in Table 1 . The computations were carried to the first three terms for both types of series. The various $k-u$ values, representing typical combinations in different ranges, were picked from the table of elliptic integrals [2], so that the "exact" values of the functions are known and that the relative accuracies of the two types of series may be compared. The percentage errors are computed to the nearest .01. It is seen that the $k$-series are nearly always more accurate than the corresponding $u$-series, particularly at larger $u$-values.

Transport Division

The Boeing Company

Renton, Washington

1. H. Bateman, Higher Transcendenlal Functions, v. II., McGraw-Hill Book Company, New York, 1953, p. 344 .

2. E. JAhNKE \& F. E. Emde, Table of Functions, Fourth Edition, Dover, New York, 1945. 